\title{
Photonic Eigenmodes in a Photonic Crystal Membrane
}

\author{
E. Ya. Glushko, ${ }^{1}$ O. E. Glushko, ${ }^{1,2}$ and L. A. Karachevtseva ${ }^{1}$ \\ ${ }^{1}$ Institute of Semiconductor Physics of NAS of Ukraine, Nauki Prsp., 45, 03028 Kiev, Ukraine \\ ${ }^{2}$ Institute of Physics, University of Leoben, Franz Josef Straße 18, 8700 Leoben, Austria \\ Correspondence should be addressed to E. Ya. Glushko, eugene.glushko@scientist.com
}

Received 24 November 2011; Accepted 21 December 2011

Academic Editors: K. Hane and S. R. Restaino

Copyright ( 2012 E. Ya. Glushko et al. This is an open access article distributed under the Creative Commons Attribution License, which permits unrestricted use, distribution, and reproduction in any medium, provided the original work is properly cited.

\begin{abstract}
Photonic membranes are the most widely used kind of 2D photonic crystals in signal processing. Nevertheless, some important aspects of electromagnetic field behavior in membrane like photonic crystals (MPCs) need detail investigation. We develop the approach close to resonant coupling modes method which unites both external and intrinsic problems, in-plane and out-of-plane geometries, and resonator properties of MPC. The resonator standing modes are excited by an external source through the special inputs and may be controlled due to the nonlinear coating. Typical photonic manifestations are studied for $\mathrm{Si}_{/} / \mathrm{SiO}_{2} 2 \mathrm{D}$ membrane resonators of rectangular.
\end{abstract}

\section{Introduction}

Usually, membranes perform the task of separation during the process of selective transport of particles of a matter through the membrane channels. In photonics, the membranes are a kind of 2D photonic crystals which may be characterized as thin and wide systems ordered in both transversal directions and filtering radiation along the normal to surface direction. Many examples of membrane usage in photonics are discussed in the literature beginning from mechanical usage in mirrors and actuators [1-3] and up to laser applications. Photonic membranes transmit and reflect incident light uniting diffractional out-of-plane phenomena and the in-plane geometry interference phenomena caused by complicated inner structure of the membrane. The membrane peculiarities in reflected light angular distribution in grating spectra were well known long ago beginning with R. Wood's work [4]. Recently, the photonic bandgap manifestations in the reflectivity of periodically patterned systems were investigated experimentally and theoretically in [5-8] using a novel resonant coupling wave method (RCWM) connecting photonic bands existing for in-plane geometry of incidence with both diffractional signals in reflection and transmission. In [9] sharp resonances in the optical transmission spectra at normal incidence were observed for high-quality chalcogenide photonic crystal membranes and associated with Fano coupling between free space and the membrane-guided modes. It was shown in [9] that the membrane-guided modes near the centre of the first Brillouin zone are responsible for the main spectral features. An overview of silicon-based photonic crystals is presented in [10]. Optical effects in the thin-film 2D photonic crystals were overviewed in [11]. The gratings on thin silicon membranes fabricated in [12] exhibit clearly expressed resonant behaviour of responses that gives an opportunity for narrow bandwidth filtering applications. In [13], reported were the silica-embedded silicon photonic crystal waveguides offered an alternative to air-channelled membranes which are fully compatible with the monolithic integration. The authors show that despite the reduced refractive index contrast compared to the air membranes, the considered structures offer an operating area near the technologically important $1550 \mathrm{~nm}$ window. More complicated spiral structure was fabricated and investigated in [14], where optical properties of the three-dimensional silicon square spiral photonic crystal were considered. In [15], the extremely high-Q channels in a two-dimensional photonic crystal membrane were investigated. The controlled interaction between the membrane and a glass fiber tip in the near field of the photonic crystal was used to build a complete spatiospectral 
map of the channel resonator mode and its coupling with the fiber tip. The near-field imaging and frequency tuning of a high-Q photonic crystal membrane was investigated in [16] as well. In [17], the ultralow loss photonic circuit based on waveguides integrated into the membrane-type photonic crystal. The transmission characteristics of the system were measured and record low propagation losses in photonic crystal waveguides of were obtained. Lasing spectrum of the GaInAsP/InP membrane laser and simulated resonant spectra for a few orders of transverse modes were obtained in [18], where a room-temperature continuous-wave operation under optical pumping was demonstrated and the threshold pump power characteristics were investigated.

The existing terminology concerning photonic crystals (PhCr), photonic membranes, and photonic resonators may be expressed in the following way. The infinite 2D structures ordered in $X Y$ plane which has also infinite size in $Z$ direction may be called the photonic crystal. The photonic crystal resonator is a finite $2 \mathrm{D}$ system with perfectly smooth side walls. This detail leads to a clear expressed angular area of total internal reflection (Figure 1, yellow lines) for field closed inside the resonator. In ideal case, the system should have infinite size in $Z$ direction. The only way to excite intrinsic standing waves is to use the input prisms due to that the external beam may hit into the total internal reflection area. The photonic membrane may be treated as a thin photonic crystal and in this capacity it exhibits properties of a two-dimensional system. The transformation from a photonic membrane to the photonic membrane resonator transforms the initial 2D system into a 3D system.

In this work, we consider a development of the existing calculation method RCWM to solve the external problem for electromagnetic field in space divided by the 2D membranetype photonic crystal into two half-spaces. The Standing Wave Expansion (SWE) method in finite 2D structures is developed for in-plane geometry of incidence. The spectrum of modes trapped inside the total internal region of the $\mathrm{Si} / \mathrm{SiO}_{2}$ photonic resonator is calculated in 0 -approximation of SWE method. In out-of-plane geometry, light reflection and transmission are investigated in chosen geometry of incidence taking into account the existing in-plane modes.

\section{In-Plane Geometry: SWE Theory for Electromagnetic Field in a Finite 2D Photonic Crystal}

The SWE method uses a special way of expansion by analytically found eigenstates of two probe finite 1D photonic crystals. In SWE, the eigenvalue problem is solved separately for two crossed perpendicularly 1D probe photonic crystals and resulting 0 -approximation $2 \mathrm{D}$ basis is obtained as a direct production of separated bases. In considered case of p-polarization, the most convenient basis of functions may be built on the modes magnetic field. The mode magnetic field spatial dependence was found in [19-22]. For example,

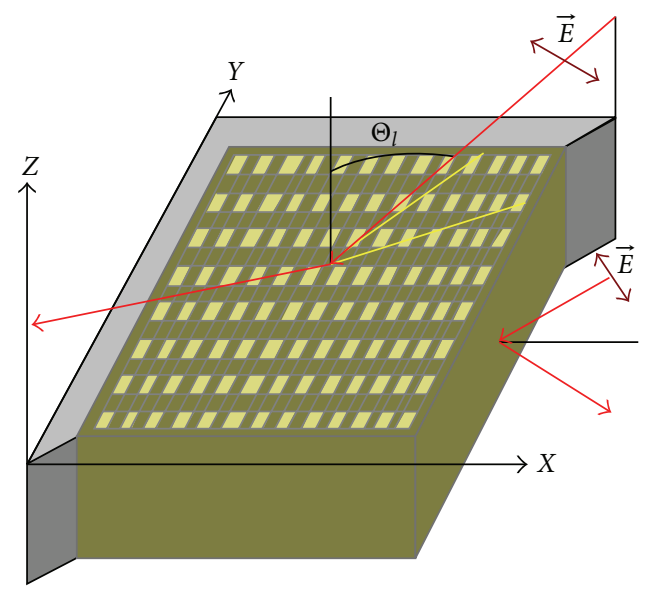

FIGURE 1: $X Y$ section of a finite $8 \times 132 \mathrm{D}$ membrane. Out-of-plane incidence: $s$-polarization, $\Theta_{l}$, external polar angle of incidence, azimuth angle $\Phi_{l}=\pi / 4$; yellow (light gray) lines, sector of total internal reflection. In-plane incidence: $p$-polarization. The input prism is not shown. Material 1, system matrix; material 2, rectangular bars in matrix; material 3, covering layer, external medium is shown by grey.

inside a $\mathrm{PhCr}$ resonator for magnetic field in an arbitrary $i j$ area we have 0 -approximation expression for mode function:

$$
\begin{aligned}
& \vec{H}_{i j}^{\mathrm{sg}}(x, y) \\
& =\vec{e}_{z} \frac{2 \cdot F_{i} \cos \left(k_{i s} x+\psi_{i s}\right)}{\left(n_{i} \sin \theta_{i}\right)} \cdot \frac{2 \cdot G_{j} \cos \left(k_{j g} y+\psi_{j g}\right)}{\left(n_{j} \sin \theta_{j}\right)}=|s, g\rangle,
\end{aligned}
$$

where $n_{i, j}$ denotes refraction index in matter of photonic crystal area $i$ or $j \cdot s, g$ enumerate the probe 1D states. $F_{i}, G_{j}$ are analytically obtained $1 \mathrm{D}$ mode amplitudes inside intrinsic areas $j, i=1, \ldots, 2 N+1$, whereas for outside areas where $i=0$ or $j=0$ cosines should transfer into $\exp \left(-k_{i s} x\right)$ or $\exp \left(-k_{j g} y\right)$, correspondingly. $\psi_{i s}, \psi_{j g}$ are analytically obtained phases in framework of 1D problem for two probe crossed photonic crystals. The wavefront orientation with respect to OX axes is given by angle $\theta_{i}=\theta_{1}$ or $\theta_{2}$ for odd or even layers of a probe $1 \mathrm{D} \mathrm{PhCr}$ with appropriate geometry [19]. The angles $\theta_{j}$ present in $y$-part of the basis function are $\pi / 2-\theta_{1}$ or $\pi / 2-\theta_{2}$ for odd or even layers, respectively. Here, the index 1 corresponds to the topologically connected matrix material 1, and index 2 denotes the embedded into the matrix ordered bars of material 2. Below, we study the rectangular photonic crystal resonator based on a $2 \mathrm{D}$ terminated binary structure consisting of the topologically connected matrix material and another one-disconnected material 2 which looks like a system of rectangular bars.

The considered medium is sectionally continued with respect to optical density. Therefore, the dielectric function $\varepsilon(x, y)$ may be carried through the derivative and we have the wave equation

$$
\frac{1}{\varepsilon(x, y)} \Delta H+\frac{\omega^{2}}{c^{2}} H=0,
$$




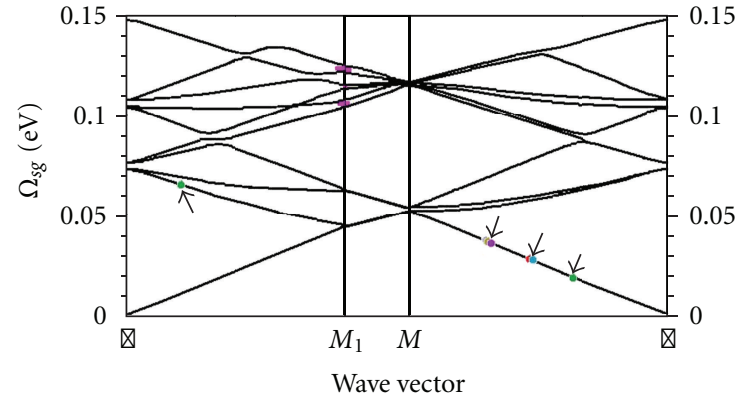

(a)

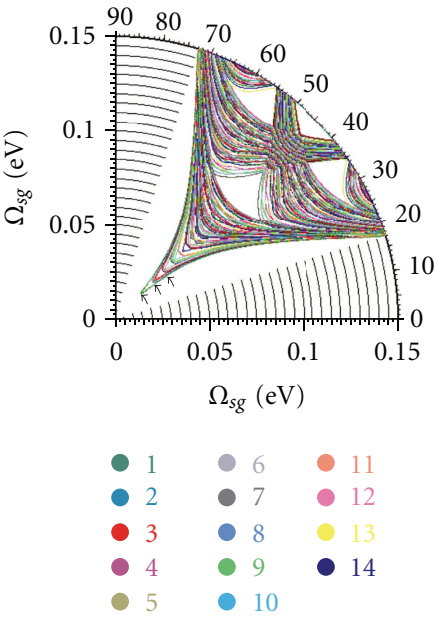

(b)

FIGURE 2: Comparison of results for finite and infinite 2D structures. Symmetry is shown in Figure 1. Elementary cell parameters: $d_{1 x}=$ $d_{1 y}=4 \mu \mathrm{m}, d_{2 x}=d_{2 y}=1 \mu \mathrm{m}$. (a) Plane wave calculation for infinite $\mathrm{Si} / \mathrm{SiO}_{2}$ photonic crystal. $\Gamma M$, diagonal $\theta_{1}=45^{\circ}$ direction, $\Gamma M_{1}$, $\theta_{1}=33.69^{\circ}$ direction. Six lowest resonator modes are shown by corresponding colour points, beginning with $\Omega_{\min } \approx 0.0197 \mathrm{eV}$ (arrows). Three lowest gaps are shown along $M_{1}$ vertical line. (b) Modes of the $8 \times 8 \mathrm{Si} / \mathrm{SiO}_{2} 2 \mathrm{D}$ rectangular lattice resonator. Angular mode diagram. Calculated by the SWE method 0-approach modes allocated in the polar diagram. Shown are 901 lowest energy existing branches in 14 colour gradation inside the total internal reflection area, energy interval $(0,0.15) \mathrm{eV}$, angular interval $17.1^{\circ}<\theta_{1}<72.9^{\circ}$, lattice period $5.0 \mu \mathrm{m}$. The corresponding lowest modes along $\Gamma M$ direction are marked by arrows.

for OZ-oriented magnetic field in the resonator. It should be taken into account that $2 \mathrm{D}$ basis $|s, g\rangle$ is composed from two separate $1 \mathrm{D}$ bases taken at angles $\theta$ and $\pi / 2-\theta:|s, g\rangle_{x y}=$ $|s(\theta)\rangle_{x} \cdot|g(\pi / 2-\theta)\rangle_{y}$. The expansion into the series gives

$$
H=\sum_{s, g} h_{s g} \cdot|s, g\rangle
$$

Then, (3) generates the system of equations for expansion amplitudes $h_{s g}$ :

$$
\left\{\begin{array}{l}
\sum_{s, g}\left[\left\langle q\left|\frac{k_{s g}^{2}}{\varepsilon(x, y)}\right| s g\right\rangle-\frac{\Omega^{2}}{c^{2}} \delta_{q, s g}\right] h_{s g}, \\
q=1, \ldots, N_{m}
\end{array}\right.
$$

where $q$ numbers states of the $2 \mathrm{D}$ basis, matrix elements $\left\langle q\left|k_{s g}^{2} / \mathcal{E}(x, y)\right| s, g\right\rangle$ mean the integral over the resonator. It should be noted that matrix elements have the analytical form in our approach as the field $H$ is described by amplitudes $F(s, i), G(g, j)$ and phases $\psi_{i s}$, $\psi_{\text {is }}$ found in 1D problem which play part of the $2 \mathrm{D}$ basis generator. The full solution is given by a multitude of eigenvalues and corresponding standing waves or resonator modes:

$$
\left\{\Omega_{q}^{2},\left(\begin{array}{c}
h_{1}^{q} \\
\vdots \\
h_{N_{m}}^{q}
\end{array}\right)\right\},
$$

where $q$ enumerates $2 \mathrm{D}$ states which are standing waves inside the total internal reflection area of the resonator, $h_{m}^{q}$ are coefficients of the $2 \mathrm{D}$ state $q$ expansion into initial basis $|s, g\rangle$ series. First, the SWE method calculation of the bandgap structure was performed for a low contrast $\mathrm{CdSe} / \mathrm{CdS}$ photonic resonator in [23].

In this paper, we consider a more contrast system-a $\mathrm{Si} / \mathrm{SiO}_{2}$ resonator of rectangular form which has $8 \times 8$ periods plus one external layer of material 3 (Figure 1). Also, the calculation was performed for an infinite $\mathrm{Si} / \mathrm{SiO}_{2}$ photonic crystal with the same symmetry by using the plane wave expansion method. The $\mathrm{PhCr}$ lattice has the rectangular symmetry (Figure 1), and bars of material 2 have also rectangular form: $d_{2 x}=d_{2 y}=1.0 \mu \mathrm{m}$ and for material 1 voids $d_{1 x}=d_{1 y}=4 \mu \mathrm{m}$. In Figure 2(a), the results calculated for the direction of symmetry $\Gamma M$ and nonsymmetry directions $k_{x}=1.5 k_{y} \Gamma M_{1}$ and $M M_{1}$ are shown. The calculation shows that gaps exist only along the $\Gamma M_{1}$ direction. Three lowest gaps are shown by arrows along the $M_{1}$ vertical line: between fourth-fifth (of width $0.025 \mathrm{eV}$ ), sixth-seventh $(0.012 \mathrm{eV})$, and ninth-tenth $(0.030 \mathrm{eV})$ Brillouin zones in the extended scheme. The resonator property to trap the wave inside is exhibited in the angular area near the diagonal $\theta_{1}=\pi / 4$ direction $(\Gamma M)$. Six lowest trapped modes taken from SWE calculation for a finite resonator are plotted at the $\Gamma M$ branch (colour points). In Figure 2(b), the mode structure calculated in 0-approximation of SWE [20] for a finite $\mathrm{PhCr}$ resonator is shown in polar coordinate system for the first 901 standing waves with accuracy $\approx 0.001$. The energy interval $(0,0.15) \mathrm{eV}$, the alternating $2 \mathrm{D}$ mode branches are shown in 14-colour scale. The mode branches in the total internal reflection area $\left(\Theta_{\text {total }}, \pi / 2-\Theta_{\text {total }}\right)$ of the resonator under study are allocated symmetrically near the bisector $\theta_{1}=\pi / 4$ and they are doubly degenerated due to the rectangular symmetry of the system. The same above-mentioned lowest resonator modes are marked by 
arrows beginning with $\Omega_{\min } \approx 0.02 \mathrm{eV}$ (Figure $2(\mathrm{~b})$ ). When $N$ increase to infinity then $\Omega_{\text {min }} \rightarrow 0$. The direction $\Gamma M_{1}$ $\left(\theta_{1}=33.69^{\circ}\right)$ in the resonator contains a gap in area of low frequencies, and the band of states begins with $\Omega_{\min } \approx$ $0.065 \mathrm{eV}$. It is caused by the fact that each wave propagating inside the resonator at an arbitrary angle $\theta$ is mixing by the resonator with the $(\pi / 2-\theta)$ wave that makes the selection rule for permitted standing waves more rigid. Therefore, the states existing in an infinite $\mathrm{PhCr}$ may be forbidden in a resonator: the first permitted resonator state arises for the $\Gamma M_{1}$ direction at $0.065 \mathrm{eV}$ and is situated on the second photonic branch in the scheme of reduced zones shown in Figure 2(a) (left arrow).

Our SWE calculations give a smooth transformation of the considered 2D mode structure from 2D structure shown in Figure 2(b) to the 1D one shown in Figure 3 if $d_{1 y}$ or $d_{2 y}$ increase in accordance with real transformation of a $2 \mathrm{D}$ resonator into a $1 \mathrm{D}$ one. The spectrum has the discrete character due to finite size of the resonator. Instead of the continuous multitude of electromagnetic eigenstates distributed along the modal surface, we have got several angle-depended mode lines. To compare the results obtained for 1D and 2D systems we have studied two 1D structures which have close parameters $d_{1}$ and $d_{2}$ and may be considered in some sense as a parent structure for the considered above $2 \mathrm{D}$ resonator [20]. The modes of the $1 \mathrm{D}$ 8 -period $\mathrm{Si} / \mathrm{SiO}_{2}$ resonator calculated by the transfer matrix method are shown in Figure 3 for the resonator total internal reflection area $17.1^{\circ}<\theta_{\text {total }}<72.9^{\circ}$ in energy interval $(0$, $0.5) \mathrm{eV}$. Geometric parameters were taken close to that for considered above 2D system: matrix material layers $d_{1}=$ $4.0 \mu \mathrm{m}$, layer thickness $d_{2}=1.0 \mu \mathrm{m}$ (glass layers). The material of covering layer was taken coinciding with the matrix: material $3=$ material 1 both for $2 \mathrm{D}$ resonator and $1 \mathrm{D}$ $\mathrm{PhCr}$. Due to the considerable optical contrast of the binary system under consideration the waveguide-type [23] angular area is present (shown by red), and the band states belonging to the transmitted type are shown by violet. The angular boundary between these two types of states passes at $\theta_{1} \approx$ $26.2^{\circ}$. The lowest $1 \mathrm{D}$ state at $\theta_{1}=\pi / 4$ is $\omega_{\min } \approx 0.014 \mathrm{eV}$ that to average $\Omega_{\min } \approx \sqrt{2} \omega_{\min }$ [20]. The considerable gaps arise far inside the total internal reflection area. Local or surface modes detached from bands in transmitted and waveguide areas are shown by blue and light blue. Upper restriction $1.272 \mathrm{rad}$ chosen for angular area is caused by the demand $\pi / 2-\Theta_{\text {total }}$ which is important for $2 \mathrm{D}$ resonators of chosen geometry.

Below we will consider the method to solve the united external-intrinsic problem for a finite 2D photonic crystal membrane.

\section{Uniting In-Plane and Out-of-Plane Geometries: The In-plane Modes Contribution Into Reflection}

The typical geometry of an external problem for a limited $2 \mathrm{D}$ membrane is shown in Figure 1. Two principally different angular areas exist for external incidence. First is when the

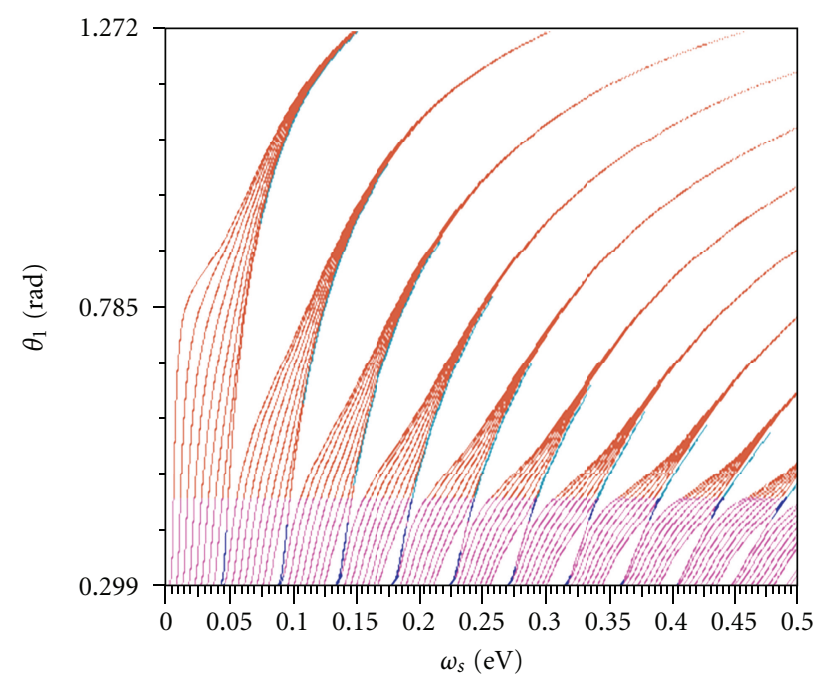

Figure 3: The modes of a $1 \mathrm{D} 8$ period $\mathrm{Si} / \mathrm{SiO}_{2}$ resonator calculated in the transfer matrix method. Shown are lowest existing branches in the total internal reflection area $17.1^{\circ}<\theta_{\text {total }}<72.9^{\circ}$ of the resonator. Extended waves (area 17.1 $1^{\circ}<\theta_{1}<26.2^{\circ}$ ) are shown by violet, waveguide-type waves $\left(\theta_{1}>26.2^{\circ}\right.$ are shown by red, detached local (surface) modes are shown by blue and light blue (waveguide area). Energy interval $(0,0.5) \mathrm{eV}, s$ enumerates $1 \mathrm{D}$ modes, layer thickness: $d_{1}=4.0 \mu \mathrm{m} d_{2}=1.0 \mu \mathrm{m}$ (glass layers).

beam hits into the sector of standing waves between the two yellow lines inside the total internal reflection of the resonator. For these directions, the membrane may be treated as an effective medium where the external wave excites the eigenmodes of the resonator. Another case arises for all directions of incidence outside the sector shown in Figure 1, and the incident wave excites extended waves in the membrane instead of eigenmodes.

We concentrate our efforts on the first regime of outof-plane incidence when azimuth angle is taken $\Phi_{l}=\pi / 4$ (Figure 1, bisector plane). Under consideration is the spolarized wave incident at the angle $\Theta_{l}$ on the top surface of the membrane. Inside the resonator, this wave excites standing waves (resonator eigenmodes) which have the character of pure p-polarized waves. In the lower semispace field transforms into s-polarized wave again.

The SWE method gives the full solution (5) for $\mathrm{p}$ polarized field inside the resonator. In terms of effective medium the solution (5) determines totally the optical characteristics of the membrane which divides total space into upper semispace and the lower one. The given frequency of incident wave $\omega$ mixes with the in-plane discrete spectrum $\Omega_{q}$ and produces $z$-component of field inside the membrane:

$$
k_{z}=\frac{\sqrt{\omega^{2}-\Omega_{q}^{2}}}{c} .
$$

The wave vector projection $k_{z}$ becomes imaginary for low frequency incident waves, and, therefore, $z$-component of field has only decaying tails inside the PCM under consideration. If wave frequencies $\omega$ become higher than $\Omega_{q}$, a line of interference peaks should arise which is caused by 
field resonance in the perpendicular to the film direction. The two-wave solution for electromagnetic field may be represented by means of the magnetic field in all three media

$$
n_{a} \vec{H}_{a}=\left(n_{a z},-n_{a x}\right) A_{a} e^{i k_{a z} z}-\left(n_{a z}, n_{a x}\right) B_{a} e^{-i k_{a z} z},
$$

where $a=\{l, m, r\}$ denotes upper half-space, membrane, and lower half-space, respectively. The amplitude $B_{r}$ for the lower half space should be taken equal to zero.

Further, boundary conditions of continuity for magnetic field at both surfaces $z=0$ and $z=L$ lead to the system of equations for unknown amplitudes. In matrix view we have

$$
\left[\begin{array}{cccc}
-h_{l x} & h_{m x} & h_{m x} & 0 \\
h_{l z} & -h_{m z} & h_{m z} & 0 \\
0 & \tilde{h}_{m x} & \tilde{h}_{m x}^{*} & -h_{r x} \\
0 & \tilde{h}_{m z} & -\tilde{h}_{m z}^{*} & h_{r z}
\end{array}\right]\left[\begin{array}{c}
B_{\mathrm{l}} \\
A_{\mathrm{m}} \\
B_{\mathrm{m}} \\
A_{\mathrm{r}}
\end{array}\right]=A_{l}\left[\begin{array}{c}
h_{l x} \\
h_{l z} \\
0 \\
0
\end{array}\right],
$$

where for all indices $a$ shorthand notations are made $h_{a x}=$ $n_{a x} / n_{a}, h_{a z}=n_{a z} / n_{a}$, and $\tilde{h}_{a x, z}=h_{a x, z} e^{i k_{a z} z}$. Equation (8) determines contribution in amplitudes $A_{r}^{q}$ and $B_{l}^{q}$ of field caused by a separate intrinsic mode $q$. For an optically linear system, the result may be expressed as a weighed sum taken over the basis of eigenmodes $q$. Therefore, supposing $A_{l}=1$, we have for reflection and transmission

$$
R=\frac{1}{M} \sum_{q=1}^{M}\left|B_{l}^{q}\right|^{2}, \quad T=\frac{1}{M} \sum_{q=1}^{M}\left|A_{r}^{q}\right|^{2},
$$

where $M$ is basis length and $q$ enumerates ordered 2D basis functions $|q\rangle$ calculated in SWE method at given azimuth angle $\Phi_{l}$. In previous section, we calculate $2 \mathrm{D}$ spectrum of intrinsic modes for in-plane geometry of incidence (Figures 1 and $3(\mathrm{~b})$ ) at $\Phi_{l}=\pi / 4$. For this bisect direction, it begins starting from $0.0197 \mathrm{eV}$ and includes 901 states in energy interval $(0,0.15) \mathrm{eV}$. In Figure 4 , the transmission versus frequency dependence for a thin $\mathrm{PhCr}$ membrane $L=50 \mu \mathrm{m}$ is shown for five $\Phi_{l}$ values in interval beginning with the almost normal incidence angle 6 grad and up to $54 \mathrm{grad}$. For all angles of incidence, the every dense group of modal states (Figure 4, bar graph 1) contributes to the transmission increase. Much less increasing or even drop is observed in the frequency regions between the peaks of the DOS. Light interference caused by the membrane effective medium exhibits itself in area of energies beginning with $0.15 \mathrm{eV}$ as small beating on the envelope dependence of transmission. Further, they became more clearly expressed beginning with $0.4 \mathrm{eV}$. Depending on the membrane thickness, the density of resonances may be lesser or higher. The transmission dependence for a thick PCM $L=100 \mu \mathrm{m}$ exhibits twice higher density of resonances beginning with $0.4 \mathrm{eV}$ than density shown in Figure 4. For a thick PCM, at each angle of incidence $\Theta_{l}$ the big density of resonances creates the envelope line showing results close to that may be obtained for a nonperfect thick 2D photonic macroporous silicon structure [24]. A more detailed analysis of resonances nature will be made elsewhere. In the inset (Figure 4), the contribution of a solitary phonon polariton mode

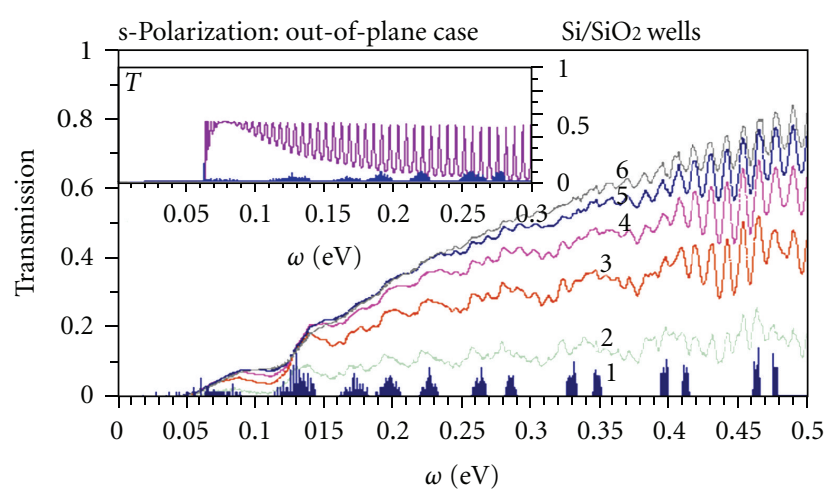

FIGURE 4: Contribution of resonator's modes into the light transmission through the membrane. SWE and coupled wave method calculation for finite $8 \times 8 \mathrm{Si} / \mathrm{SiO}_{2}$ photonic membrane. Contribution of resonator's modes $\Omega_{q}>0.02$. Incidence in the diagonal $\theta_{1}=\pi / 4(\Gamma M)$ direction. Elementary cell parameters: $d_{1 x}=d_{1 y}=4 \mu \mathrm{m}, d_{2 x}=d_{2 y}=1 \mu \mathrm{m}$. Shown is transmission in energy interval $(0,0.5) \mathrm{eV} .1$, normalized DOS diagram. Angles of incidence. 2: $\Theta_{l}=6^{\circ} ; 3: \Theta_{l}=18^{\circ} ; 4: \Theta_{l}=30^{\circ} ; 5: \Theta_{l}=42^{\circ} ; 6$ : $\Theta_{l}=54^{\circ}$. The membrane thickness, $L=50 \mu \mathrm{m}$. Inset: contribution of the phonon polariton mode $\Omega_{\mathrm{ph}}=0.0629 \mathrm{eV}, L=100 \mu \mathrm{m}$, $\Theta_{l}=36^{\circ}$.

$\Omega_{\mathrm{ph}}=0.0629 \mathrm{eV}, \Theta_{l}=36^{\circ}$ added with weight number $=$ 2000 to the found 901 pure electromagnetic modes is plotted.

In considered approach, the photonic crystal membrane is treating in spirit of an effective medium describing by a multitude of in-plane resonant eigenmodes. Another (nonresonator) case arises if azimuth angle $\Phi_{l}$ lies outside the sector of total internal reflection area of the resonator. Therefore, this case needs another approach which will be considered in another work. It should be noted that in the SWE method developed in present paper the information about direction of initial waves remains in modal numbers. Second, the expansion procedure in SWE is carried out by standing waves which have practically zero amplitudes outside the resonator.

\section{Summary}

Here, we develop the approach uniting both external and intrinsic problems, in-plane and out-of-plane geometries, and resonator properties of PCM. The resonator in-plane standing modes can be excited by an external source through the special inputs and may be controlled due to their nonlinear properties. Also, we have considered light transmission in out-of-plane geometry for rectangular $2 \mathrm{D}$ photonic membrane resonators where the incident wave may excite the trapped standing modes. Finally, it should be noted that the proposed SWE method for finite resonators uses open boundary conditions and may be adapted for any symmetry of the lattice as well as for any shape of material 2 bars in matrix material. 


\section{References}

[1] D. K. Marker and C. H. Jenkins, "Surface precision of optical membranes with curvature," Optics Express, vol. 1, no. 11, pp. 324-331, 1997.

[2] H. M. Dyson, R. M. Sharples, N. A. Dipper, and G. V. Vdovin, "Cryogenic wavefront correction using membrane deformable mirrors," Optics Express, vol. 8, no. 1, pp. 17-26, 2001.

[3] C. Paterson, I. Munro, and J. C. Dainty, "A low cost adaptive optics system using a membrane mirror," Optics Express, vol. 6, no. 9, pp. 175-185, 2000.

[4] R. W. Wood, "On a remarkable case of uneven distribution of light in a diffraction grating spectrum," Philysophical Magazine, vol. 4, pp. 396-402, 1902.

[5] V. N. Astratov, D. M. Whittaker, I. S. Culshaw et al., "Photonic band-structure effects in the reflectivity of periodically patterned waveguides," Physical Review B, vol. 60, no. 24, pp. R16255-R16258, 1999.

[6] V. N. Astratov, I. S. Culshaw, R. M. Stevenson et al., "Resonant coupling of near-infrared radiation to photonic band structure waveguides," Journal of Lightwave Technology, vol. 17, no. 11, pp. 2050-2057, 1999.

[7] D. Cassagne, C. Jouanin, and D. Bertho, "Hexagonal photonicband-gap structures," Physical Review B, vol. 53, no. 11, pp. 7134-7142, 1996.

[8] V. M. Fitio and Y. V. Bobitski, "Diffraction analysis by periodic structures using a method of coupled waves," Opto-Electronics Review, vol. 13, no. 4, pp. 331-339, 2005.

[9] C. Grillet, D. Freeman, B. Luther-Davies et al., "Characterization and modeling of Fano resonances in chalcogenide photonic crystal membranes," Optics Express, vol. 14, no. 1, pp. 369-376, 2006.

[10] A. Birner, R. B. Wehrspohn, U. M. Gösele, and K. Busch, "Silicon-based photonic crystals," Advanced Materials, vol. 13, no. 6, pp. 377-388, 2001.

[11] M. Kellegoz, I. Ozkan, and M. S. Kilickaya, "Performance effects of proton exchange membrane fuel cell at various operating temperatures," Journal of Optoelectronics and Advanced Materials, vol. 10, no. 2, pp. 369-372, 2008.

[12] Y. Wang, Y. Kanamori, J. Ye, H. Sameshima, and K. Hane, "Fabrication and characterization of nanoscale resonant gratings on thin silicon membrane," Optics Express, vol. 17, no. 7, pp. 4938-4943, 2009.

[13] T. P. White, L. O’Faolain, J. Li, L. C. Andreani, and T. F. Krauss, "Silica-embedded silicon photonic crystal waveguides," Optics Express, vol. 16, no. 21, pp. 17076-17081, 2008.

[14] S. R. Kennedy, M. J. Brett, H. Miguez, O. Toader, and S. John, "Optical properties of a three-dimensional silicon square spiral photonic crystal," Photonics and Nanostructures, vol. 1, no. 1, pp. 37-42, 2003.

[15] K. H. Hwang and G. H. Song, "Design of a high-Q channel add-drop multiplexer based on the two-dimensional photonic-crystal membrane structure," Optics Express, vol. 13, no. 6, pp. 1948-1957, 2005.

[16] S. Mujumdar, A. F. Koenderink, T. Sünner et al., "Near-field imaging and frequency tuning of a high Q photonic crystal membrane microcavity," Optics Express, vol. 15, no. 25, pp. 17214-17220, 2007.

[17] S. J. McNab, N. Moll, and Y. A. Vlasov, "Ultra-low loss photonic integrated circuit with membrane-type photonic crystal waveguides," Optics Express, vol. 11, no. 22, pp. 29272939, 2003.

[18] T. Maruyama, T. Okumura, S. Sakamoto, K. Miura, Y. Nishimoto, and S. Arai, "GaInAsP/InP membrane BH-DFB lasers directly bonded on SOI substrate," Optics Express, vol. 14, no. 18, pp. 8184-8188, 2006.

[19] E. Y. Glushko, "Switching of electromagnetic eigenwaves in metastructures," in Photonic Crystal Materials and Devices, vol. 6989 of Proceedings of SPIE, 2008.

[20] E. Y. Glushko, A. E. Glushko, V. N. Evteev, and A. N. Stepanyuk, "Electromagnetic eigenwaves in metastructures: perturbation theory method," in Nanophotonics II, vol. 6988 of Proceedings of SPIE, p. 118, 2008.

[21] E. Ya. Glushko, "All-optical signal processing in photonic structures with nonlinearity," Optics Communications, vol. 247, no. 4-6, pp. 275-280, 2005.

[22] E. Ya. Glushko, Optics Express 18, p. 3071, 2010.

[23] E. Y. Glushko, A. E. Glushko, V. N. Evteev, and A. N. Stepanyuk, "All-optical signal processing based on trapped modes of a photonic crystal resonator," in 3rd Nonlinear Optics and Applications, vol. 7354 of Proceedings of SPIE, 2009.

[24] L. A. Karachevtseva, O. O. Lytvynenko, E. O. Malovichko, O. J. Stronska, E. V. Busaneva, and O. D. Gorchinsky, "Optical transmittance of 2D macroporous silicon structures," Semiconductor Physics, vol. 4, no. 4, pp. 347-351, 2001. 

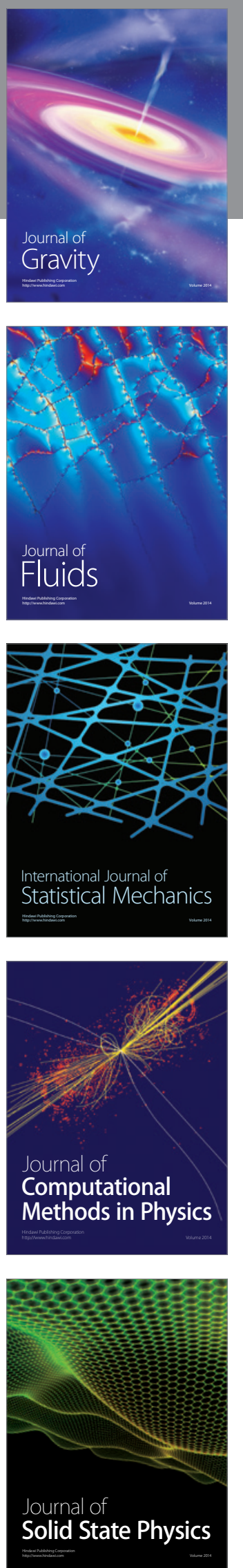
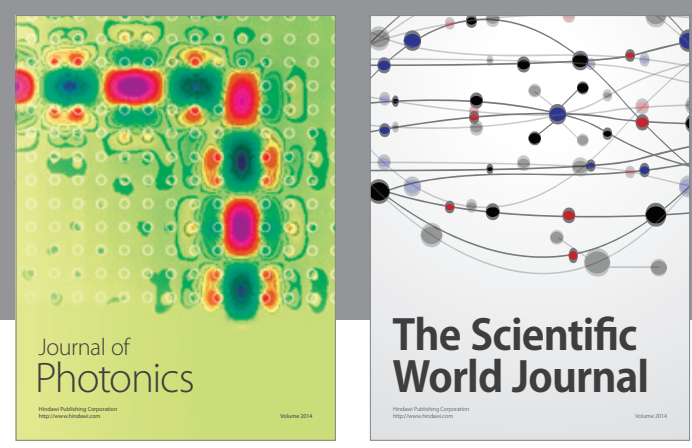

The Scientific World Journal

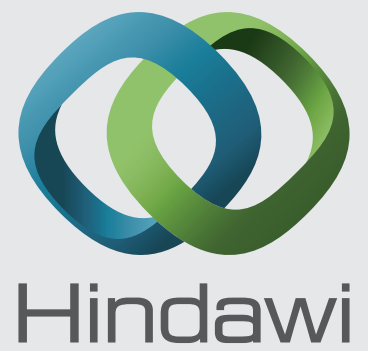

Submit your manuscripts at http://www.hindawi.com
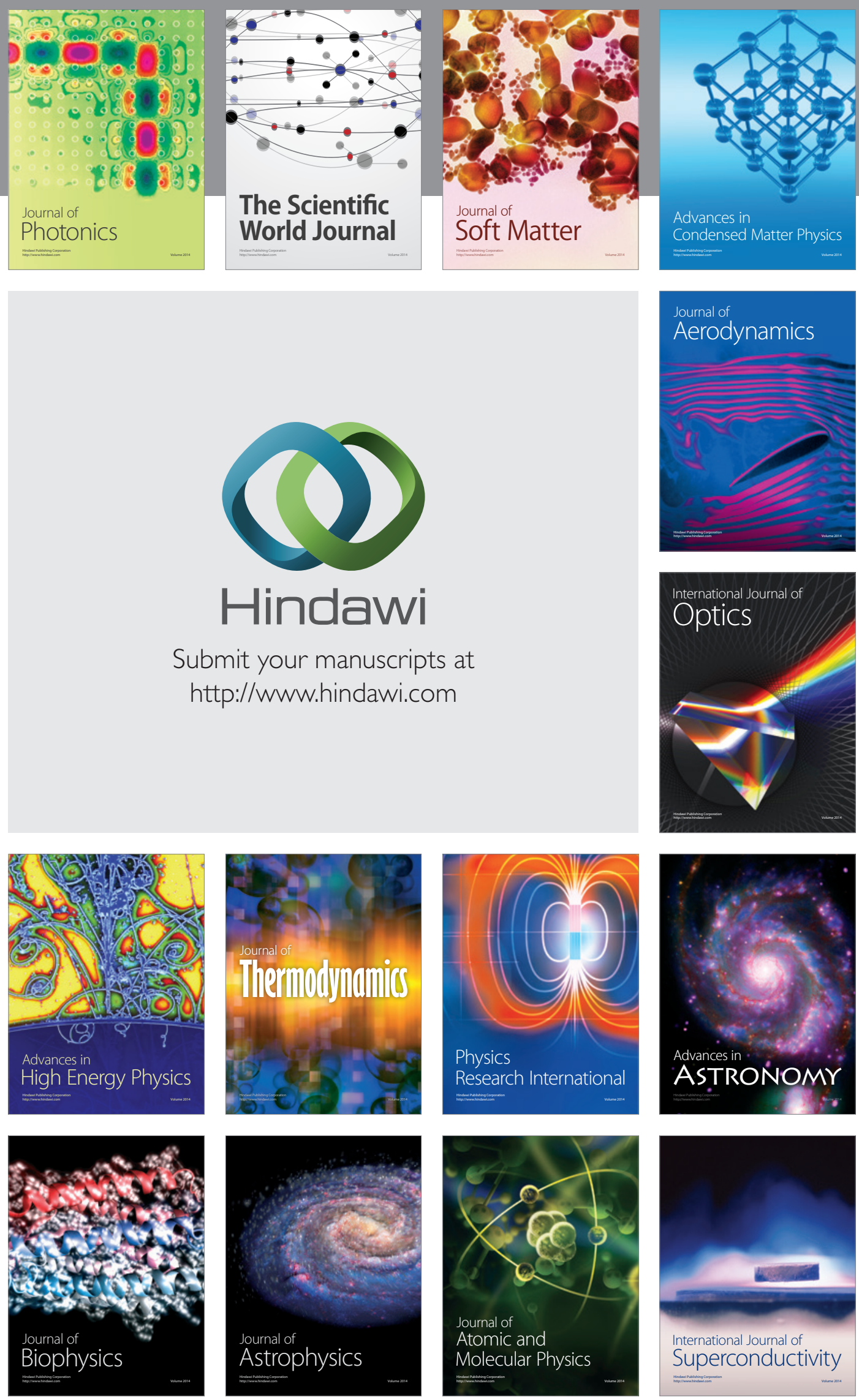
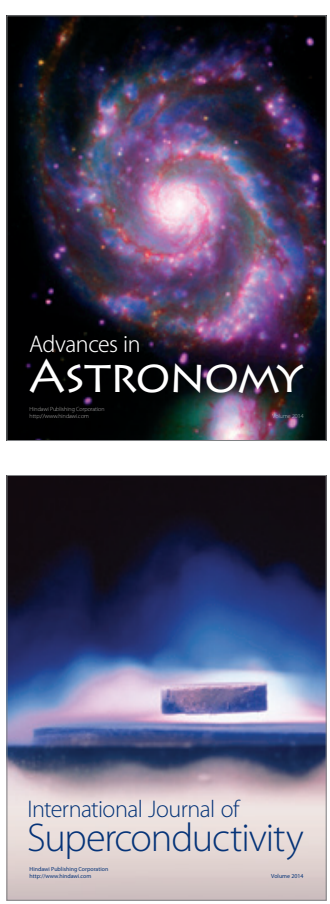